\title{
Intercellular Junctions in the Human Vascular Stria and Spiral Ligament
}

\author{
Masayoshi Tachibana, Susumu Nakae, Mitsuo Machino, \\ Hiroyuki Morioka and Osamu Mizukoshi \\ (Kyoto Prefectural University of Medicine)
}

\begin{abstract}
The intercellular junctions of the vascular stria and spiral ligament in the human cochlea were examined by means of the freeze-fracture technique.

It appeared that the vascular stria and the spiral ligament of the human shared common anatomical characteristics with that of the guinea pig as far as the intercellular junctions are concerned and we could not find any reason not to assume that the electrophysiological nature of the vascular stria of the human is basically identical to that of the guinea pig.

The tight junctions observed at the apical portion of the marginal cell could be classified as "intermediate to tight". The junctions observed between the basal cells were classified as"very tight". Thus the vascular stria is barred from both endolymph and perilymphatic tissue (spiral ligament) by the tight junction.

Virtually all the component cells of the vascular stria and the spiral ligament seemed to be interconnected by gap junctions. These cells might share a common intracellular ionic environment.
\end{abstract}

The junctional complexes in the vascular and spiral ligament have attracted the interest of many researchers since steep ionic gradient and static potential between endolymph and perilymph were demonstrated (Smith et al, 1954; Bekesy, 1952). They have been repeatedly studied at the electronmicroscopic level (Jahnke, 1974 and 1975; Tachibana, 1975). However, these studies have been done so far in experimental animals such as the guinea pig and the chinchilla. We thought it would be necessary to study the junctional complex in human tissue in order to make clinical application of the findings in experimental animals.

\section{MATERIALS AND METHODS}

The cochlea from three autopsy subjects were studied. Autopsy subjects consisted of three persons who suffered from hypertention and died of causes irrelevant to the hearing organ. The fixative containing $2.5 \%$ glutaraldehyde, $0.1 \mathrm{M}$ phosphate buffer $(\mathrm{pH} 7.4)$ was injected into the tympanic cavity through the tympanic membrane immediately after the judgement of death, and the temporal bone was extirpated within three hours. The 
bony cochlea was opened in the fixative to allow an adequate penetration of the fixative. Then the lateral walls of the cochlea were dissected out and fixed in the same fixative for at least two hours. The specimens were then transferred into $45 \%$ glycerol in saline for overnight, mounted in specimen holders, frozen in liquid nitrogen and fractured in an appratus developed by ourselves. The fractured surface was shadowed with a platinum-carbon mixture at a $45^{\circ}$ angle followed by evaporation of carbon at the normal angle to the planc. The replicas were removed from the specimen with household bleach and washed with distilled water. The cleaned replicas were picked up on 200-mesh grids and examined.

\section{Tight Junctions}

\section{RESULTS}

Tight junctions were observed in the vascular stria but not in the spiral ligament. Between the marginal cells strands of tight junctions were found adjacent to the endolymphatic space. At the superficial level the strands ran parallel to the free surface of the marginal cells to compose the layers which did not exceed nine; most strands were interconnected to form a network. At the deeper level the strand formed a coarser network and some strands stretched towards a basal direction (Fig 1).

Tight junctions observed between the superficial and deeper layers of the basal cells were well developed (Fig 2). Some areas surrounded by strands of tight junction contained particles of the gap junction (Fig 3)

\section{Gap Junctions}

As for the gap junction in the vascular stria and the spiral ligament, again we could not find any essential difference between the guinea pig and the human. So far gap junctions were observed between the marginal cells at their basal portion, between the basal cells surrounded by strands of tight junction (Fig 3), between the spiral ligament cells (Fig 4). Among these, gap junctions between the spiral ligament cells occured most frequently.

\section{DISCUSSION}

The present study shows that it is most likely that at least in a qualitive sense the human vascular stria and spiral ligament share the anatomical characteristics of the guinea pigs as far as the junctional complexes are concerned. We could not find any reason not to assume that the electrophysiological nature of the vascular stria of the human is basically identical to that of the guinea pig. This encourages us to carry out further electrophysiological study of the guinea pig vascular stria in order to understand human hearing.

Epithelia vary with respect to transepithlial permeability or resistance. It is found that the developmental degree of tight junction (number of strands and/or depth of junction) correlates with the resistance. Thus the epithelia or tight jumctions could be separated into several categories; very leaky, leaky, intermediate, intermediate to tight, tight and very tight junctions (Claude and Goodenough, 1973). In this term tight junctions revealed at two kinds of mammals could be classified in the same category at each different site of the vascular stria of the species; guinea pig or human (Tachibana, 1977)

The tight junctions observed at the apical portions of lining marginal cells could be classified as "intermediate to tight." Endolymph, which is believed to be secreted from the marginal cells, contains an extremely high concentration of potassium and a low 


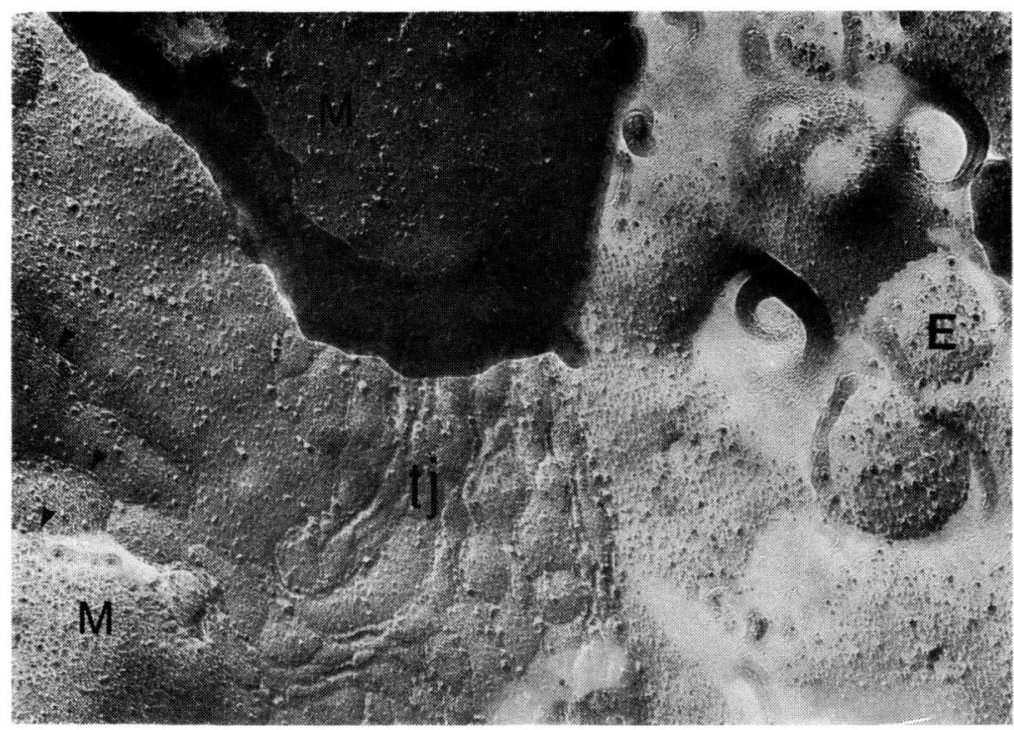

Fig 1. Tight junction ( $\mathrm{t} j$ ) between marginal cel's. At the deepe $i$ level the strands strech towards a basal direction (arrows). E:(endolymphatic space, M:marginal cel s. $\times 68,000$

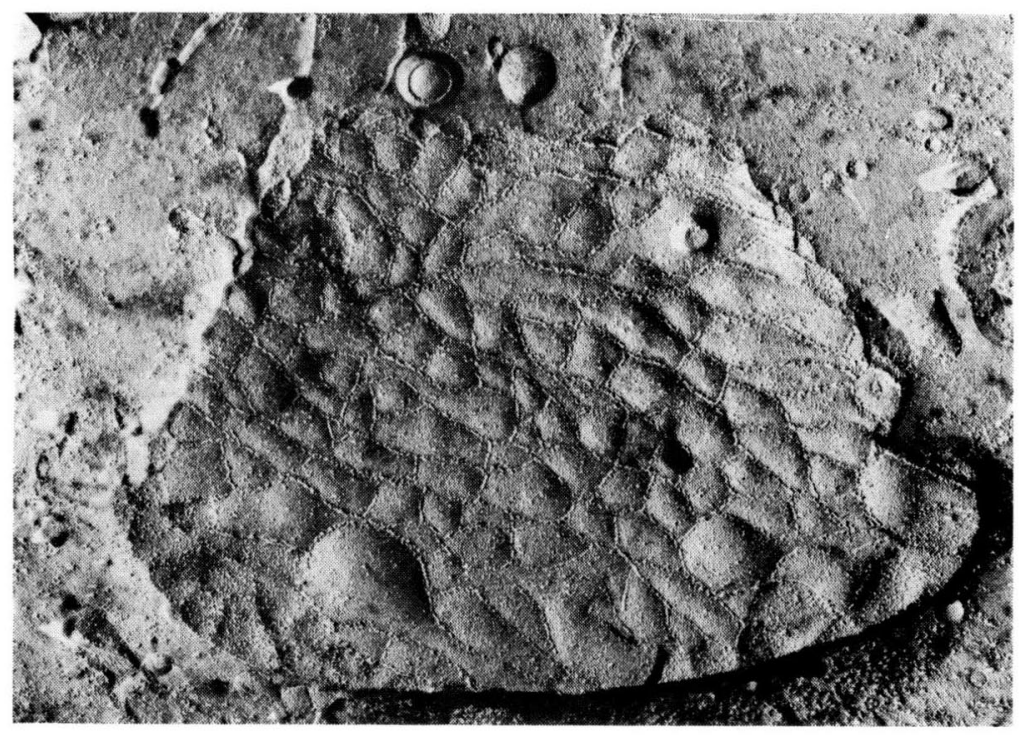

Fig. 2. Tight junction between the basal celis. $\times 34,000$ 


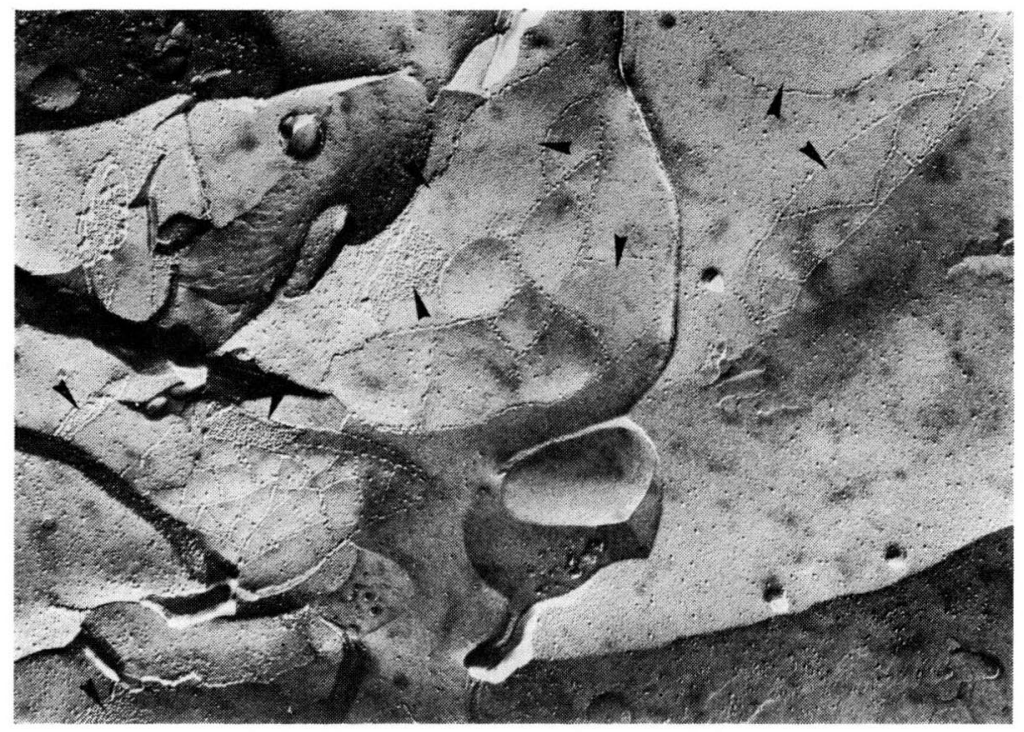

Fig. 3. The area surrounded by the strands of tight junction between the basal cells is occupied by particles of gap junction (arrows) $\times 42,000$

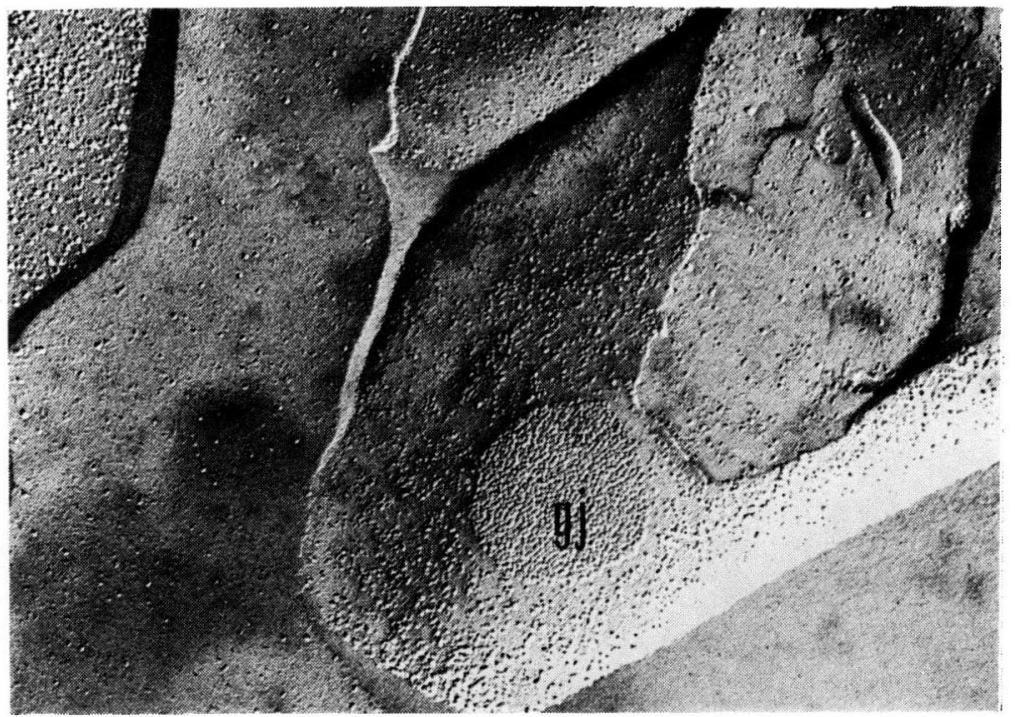

Fig. 4. A gap jnnction $(\mathrm{gj})$ between the spiral ligament cells. $\times 60,000$ 
concentration of sodium and calcium compared to ordiary extracellular fluids (Smith, Lowry and $\mathrm{Wu}, 1954)$. These ionic concentrations can be maintained only when physiologic "pumping" works, presumably in the marginal cells, which naturally requires metabolic energy. The tight junction barrier observed at the apical portion of the marginal cells may function to minimize the leakage of endolymphatic potassium to spare energy expenditure. Molecules injected into the endolymph are reported not to penetrate into the vascular stria (Yamamoto and Nakai, 1964). Another function of this barrier may be to protect the endolymph from noxious malecules brought in via the vessels. Tracer experiments support this concept, the intravascullarly injected horse-radish peroxidase does permeate the extracellular space but never reaches the endolymph (Duvall, Quick and Sutherland, 1971).

The tight junction observed between the basal cells was classified as "very tight" (Claude and Goodenough, 1973). This indicates a strong barrier between the vascular stria and the spiral ligament, the perilymphatic tissue and vice versa. Again this concept is supported by tracer experiments (Duvall and Sutherland, 1971). Thus, the vascular stria is likely to be protected from noxious molecules brought into the perilymph via vessels, the cochlear aquaeduct, the round window or any other route unless they are cell membrane permeable. Also it is not surprising that the ionic or other characteristic of the extracellular fluid of the spiral ligament; the perilymphatic tissue differs from that of the vascular stria; the endolymphatic tissue.

It appears that virtually all the component cells of the vascular stria and the spiral ligament cells are interconnected by gap junctions, aside from the intermediate cell which we could not identify in this study. These findings are already well documented in the guinea pig and in the chinchilla. The gap junction is known to connect the adjacent cells ionically. Thus it is most likely that all the component cells of the vascular stria and the spiral ligament cells share the common intracellular ionic environment.

\section{REFERENCES}

Bekesy G, Von:DG resitng potential inside the cochlear partition. J Acoust Soc Amer 24: 72-76 (1952).

Glaude, P., and D.A. Goodenough: Fracture face of zonulae occludentes from "tight" and "leaky" epithelia. J Cell Biol 58: 390-400 (1973).

Duvall, A.J., C.A. Quick and C.R. Sutherland: Horseradish peroxidase in the lateral cochlear wall. Arch Otolaryng. 93: 304-316 (1971)

Duvall, A.J. and C. R. Sutherland : Cochlear transport of horseradich peroxidase. Ann Otol 18: 705-713 (1972).

Janke, K.: The fine structure of freeze-fractured intercellular junctions on the guinea pig inner ear. Acta Otolaryng. Suppl 336 (1976).

Reale, E., L. Luciano, K. Franke, E. Pannese, G. Wermbter. and S. Iurato: Intercellular junctions in the vascular stria and spiral ligament. J
Ultrastruct Res 53: 284-297 (1975).

Smith G.A., O.H. Lowry and M.L.Wu: Electrolytes of labyrinthine fluids. Laryngoscope 64: 141-153 (1954).

Tachibana, M.: Cochlear structure as revealed by freeze-fracture and ultrahistochemistry for mucosubstance. J. Otolaryng. Jap 80: 39-50 (1977).

Winther, F.O.: The permeability of the guinea pig cochlear capillaries to horseradish peroxidase. Z. Zellforsh 114: 193-202 (1971).

Yamamoto, K. and Y. Nakai: Electronmicroscopic studies on the functions of the stria vascularis and the spiral ligament in the inner ear. Ann Otol 73: 332-347 (1964).

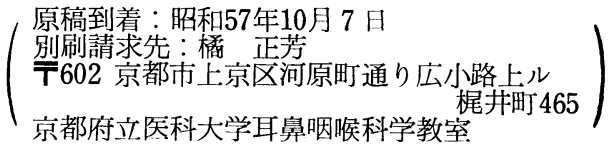

